\title{
The effect of group therapy for Japanese university students with high-functioning autism spectrum disorder and attention deficit/hyperactivity disorder
}

\author{
Yuko Furuhashi Shizuoka University, Japan
}

\section{Introduction}

Autism spectrum disorder (ASD) and attention deficit/hyperactivity disorder (ADHD) are both neurodevelopmental disorders with onset during childhood. The symptoms of both conditions frequently co-occur. However, there are few studies on treatments for young adults with ASD + ADHD. The aim of this study is to examine to the effectiveness of group therapy for university students with high-functioning ASD + ADHD.

\section{Methods}

All patients were recruited through the Health care center of Shizuoka University. The group therapy programs consisted of cognitive behavioral therapy and mindfulness therapy. Group therapy sessions were held biweekly for 24 weeks by a clinical psychologist and a psychiatrist. The Beck Depression Inventory (BDI), the Clinical Global Impression Scale (CGI-S), the Beck Anxiety Inventory (BAI), the Rosenberg self-Esteem Scale (RSES), the Conners' Adult ADHD Rating Scale Self-Report (CAARS), and the AutismSpectrum Quotient (AQ) were completed upon enrolment and at 24-week follow up.

\section{Results}

There were seven participants, four males and three females, aged between 19 and 23 years. The attendance rate for all participants was over $70 \%$ during the sixmonth group therapy. Significant improvements were observed in the total scores for BDI, BAI, and RSES. On the other hand, the total scores of CGI-S, CAARS, and AQ did not significantly change.

\section{Table 1}

Comparisons baseline and endpoint

\begin{tabular}{|c|c|c|}
\hline & Baseline (0 week) & Endpoint (24 week) \\
\hline Mean CGI-S (SD) & $3.3(0.6)$ & $3.0(0.7)$ \\
\hline Mean BDI (SD) & $12.3(2.8)$ & $8.6(3.0)^{*}$ \\
\hline Mean BAI (SD) & $29.1(7.7)$ & $21.3(2.0)^{*}$ \\
\hline Mean RSES (SD) & $11.0(1.8)$ & $13.4(1.7)^{*}$ \\
\hline Mean CAARS (SD) & $68.4(5.0)$ & $66.9(8.3)$ \\
\hline Mean AQ (SD) & $33.1(3.0)$ & $32.4(2.7)$ \\
\hline
\end{tabular}

$*_{p}<0.05$ : The Wilcoxon's single-rank test

\section{Conclusion}

The results slowed significant post-intervention improvements in depressive symptoms, anxiety, and self-esteem.

These results indicated the group therapy may be effective in this population,. 